\title{
GPS observations of post-storm TEC enhancements at low latitudes
}

\author{
Ivan Kutiev ${ }^{1}$, Yuichi Otsuka ${ }^{2}$, Akinori Saito ${ }^{3}$, and Shigeto Watanabe ${ }^{4}$ \\ ${ }^{1}$ Geophysical Institute, Bulgarian Academy of Sciences, Sofia 1113, Bulgaria \\ ${ }^{2}$ Solar-Terrestrial Environment Laboratory, Nagoya University, Toyokawa, Aichi 442-8507, Japan \\ ${ }^{3}$ Graduate School of Science, Kyoto University, Kyoto 606-8502, Japan \\ ${ }^{4}$ Graduate School of Science, Hokkaido University, Sapporo 060-0810, Japan
}

(Received February 16, 2006; Revised June 15, 2006; Accepted July 3, 2006; Online published December 25, 2006)

\begin{abstract}
In a previous work (J. Geophys. Res., 110(A01308), 1-11, 2005), the authors developed an original approach to the processing of total electron content (TEC) data obtained by GPS signals from the Japan receiver network. This approach includes removing the diurnal and seasonal variation carried by 27-day medians and the solar rotation periodicity. The relative deviations of TEC from the median-from all measured locations at a given hour-were then approximated by a regression line along the main prolongation of the Japan islands, between latitudes $24^{\circ}$ and $45^{\circ} \mathrm{N}$. The two variables of the regression line, the average value at the center and the slope were obtained as a time series, and their behavior during geomagnetic storms in the period 2000-2002 were analyzed. One interesting result was the observed enhancement of TEC at the end of the recovery phase of the storms. The slope variations clearly showed that this enhancement started from the south and was interpreted as a poleward expansion of equatorial crest. In the present paper we further analyze this post-storm phenomenon, adding foF 2 data from Japanese Kokubunji and Okinawa ionosondes. We also show the latitude extension of the poleward expansion by using lat/UT contour plots. The results confirm that most of the post-storm TEC enhancements are part of the equatorial crest region which extends poleward during nighttime. In some cases, the enhanced TEC structures develop by separating from the crest region. Daytime TEC enhancements were also observed. Their structures are not confined to the equatorial crests region, but occupy the whole latitude range considered in this study. TEC post-storm enhancements were generally found to be in agreement with foF2 variations.
\end{abstract}

Key words: Low latitude ionosphere, GPS derived Total Electron Content (TEC), low latitude TEC enhancement, equatorial anomaly crests.

\section{Introduction}

There is very little mention in the published literature of post-storm enhancement of total electron content (TEC). This phenomenon has been observed by Lin et al. (2005) who, during their study of the large-scale variation in the low latitude ionosphere during the October-November 2003 geomagnetic storm, found an increase of TEC at low latitudes on 1 November, at the time when Dst recover to its pre-storm values. The daytime increase of TEC was seen to expand poleward, with a more pronounced increase in the American sector. The ion drift data from ROCSAT used in their analysis indicated a slight upward drift, but this was not enough strong to be able explain the observed enhancement. Liu et al. $(2002,2004)$ reported a marked increase of foF2 in low latitude stations 2 days following the onset of the storm. This increase following the geomagnetic storm of 14-17 July 2000, which they discussed in their 2002 publication, was observed at the low latitude ionosonde stations of Chungli $\left(13.8^{\circ} \mathrm{N}\right.$ magnetic latitude) and Wuhan $\left(19.3^{\circ} \mathrm{N}\right.$ magnetic latitude), but not at Kokubunji $\left(25.7^{\circ} \mathrm{N}\right.$ magnetic latitude). In their second paper, these authors analyzed the post-storm increase of foF 2 following the geomagnetic

Copyright (c) The Society of Geomagnetism and Earth, Planetary and Space Sciences (SGEPSS); The Seismological Society of Japan; The Volcanological Society of Japan; The Geodetic Society of Japan; The Japanese Society for Planetary Sciences; TERRAPUB storm of 6-8 April 2000 and reported that the post-storm increase, observed at the same low latitude stations, is associated with a lowering of the F layer in comparison with its position during quiet conditions.

The generation mechanism and properties of the poststorm density enhancement remain unclear. Kelley et al. (2004) provided a quantitative explanation of the appearance of the so-called storm-enhanced density (SED) at midlatitudes that resembled the structures described by Kutiev et al. (2005). SED appears during the initial phase of the storm, when a strong auroral electric field frequently penetrates into the mid- and low latitudes. During the day, the dawn-dusk electric field produces an upward $\mathrm{E} \times \mathrm{B}$ drift that lifts the plasma in the equatorial $\mathrm{F}$ region to higher altitudes, which then diffuses down along magnetic field lines to the mid-latitude $\mathrm{F}$ region. During the nighttime, the penetrating poleward (meridional) electric field drives the $\mathrm{F}$ region plasma westward. On the sunlit part of the ionosphere, this meridional electric field vanishes due to the highly conducting E layer ionization, with the result that the plasma accumulates at the dusk terminator. SED is formed as a narrow structure (plume) along the dusk meridian. While there is experimental evidence that electric fields penetrate the low latitude ionosphere during geomagnetic storms, it is still unclear how such fields appear at these latitudes when geomagnetic activity is low. The aim of the investigation 


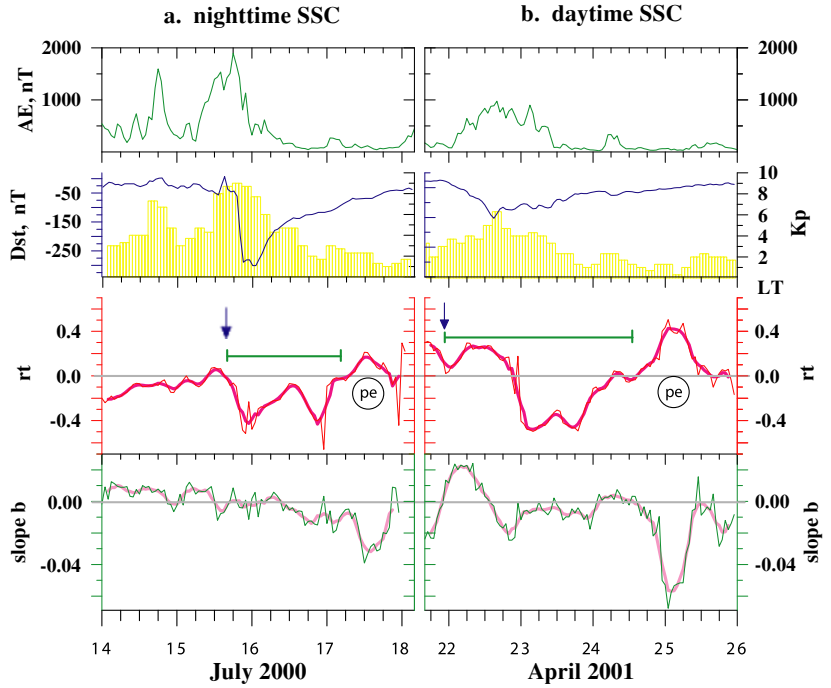

Fig. 1. Summary plots of $r t$ and the slope $b$ (bottom panel), Dst (blue line), $\mathrm{Kp}$ (yellow bars) and $\mathrm{AE}$ (top panel) during magnetic storms: 14-17 July 2000 (a) and 22-25 April 2001 (b). The day numbers on the $\mathrm{x}$-axes of the bottom panels, placed at the beginning of the days, are the same for all panels in the column. $r t$ and $b$ are representative of the latitude range $\left(26^{\circ}-45^{\circ} \mathrm{N}\right)$. The blue vertical arrows and the green horizontal bars mark the beginning of the storms and their duration. The circled letters "pe" mark the post-storm effect explained in the text.

reported here was to supplement our current body of information on this low latitude phenomenon.

Kutiev et al. (2005) studied the storm time behavior of TEC over Japan using a special manipulation technique that removed diurnal, seasonal and 27-day solar rotation periodicities in the data. The distribution of TEC storm variations over Japan was ultimately represented by a regression line taken along the main prolongation of the island. This regression line is characterized by the average value at the center and a positive gradient towards north. Instead of TEC, these researchers used the relative deviation of TEC from 27-day median, denoted as $r t$. They found that a strong enhancement of $r t$ accompanied with a large southward gradient $b$ often took place at the end of the recovery phases of geomagnetic storms. Kutiev et al. (2005) suggested that this behavior is a signature of a strong poleward expansion of equatorial crests of enhanced density. The most curious result of their investigation was that the geomagnetic activity during the event was usually very low, with a lack of any auroral or substorm activity.

\section{Data Manipulation}

Otsuka et al. (2002) described the method of obtaining the absolute TEC values from GPS signals over the Japan area. In this method, a weighted least square fitting is used to determine instrumental biases, assuming that the hourly TEC average is uniform within an area covered by a GPS receiver. The measurements from numerous locations (more than 1000 in the area) were averaged within cells that were $1.5 \times 1.5^{\circ}$ in size (geographic scale). For each cell, TEC hourly values, obtained by averaging the routine 30 s sampling-rate-TEC, were then formatted as a continuous time series during a whole year. Three years of data (20002002) were used in the analysis. A detailed description of the procedure is given by Kutiev et al. (2005). Twenty-fourhourly median values were obtained in each cell within the 27-day time window and assigned to the central 14th day. The window was then allowed to slide by 1 day, and again the new hourly medians were assigned to the next central day. By repeating the procedure, median values were obtained for each cell from 14 January 2000 to 15 December 2002. The medians were next used to calculate the relative deviations of individual TEC values from the respective medians. The relative deviation $\mathrm{TTEC}$ was defined as:

$$
\mathrm{rTEC}=\frac{\mathrm{TEC}-\mathrm{TEC}_{\mathrm{med}}}{\mathrm{TEC}_{\mathrm{med}}}
$$

The dimensionless quantity rTEC was used further in the analysis, instead of the absolute values of TEC. As has been pointed out in many ionospheric studies (see, for example, Codrescu et al., 1997; Muhtarov et al., 2002), the medians capture the average (most likely) diurnal and seasonal variations; consequently, the relative deviations reflect mainly the storm-time changes in ionospheric behavior and are suitable for comparing and analyzing data, collected during various geomagnetic storms. The basic approach of Kutiev et al. (2005) was to represent all rTEC values obtained at each hour by few average quantities and then to follow their particular behavior. For this purpose, a $4^{\circ}$-wide band was selected around the main prolongation of the Japan area $\left(24^{\circ} \mathrm{N}, 124^{\circ} \mathrm{E}\right)$ to $\left(45^{\circ} \mathrm{N}, 145^{\circ} \mathrm{E}\right)$ and only data collected inside that band were considered for analysis. The hourly rTEC values within the band area were used to calculate a regression line, described by its central value $r t c$ and slope $b$. When this approach was adopted, rTEC values in the bounded area were represented by a plane surface that was characterized by its value at the center of the plane and its slope along the main axis, tilted to $45^{\circ}$ toward the east. The sample correlation coefficient of each individual regression, which is a measure of how well the regression line approximates the rTEC values, ranged between 0.5 and 1.0 , with an average value of 0.65 .

The main feature of $r t c$ and $b$ is the absence of diurnal and seasonal variations, which are captured by the extracted medians. Kutiev et al. (2005) examined the power spectra of $r t c$ and found a well-pronounced peak at $640 \mathrm{~h}$ (26.7 days), which represented the solar rotation period. To remove this periodicity, Kutiev et al. (2005) approximated the induced variation by a Fourier wave with a main period of $640 \mathrm{~h}$ (26.7 days) and the first two harmonics. The wave approximation captured more than $30 \%$ of the $r t c$ variations. The subtracted quantity $r t=r t c-r t c w$ was the final data product used further in the storm analysis.

\section{Storm-Time TEC Behavior}

The data set with the regression parameters $r t$ and $b$ was used by Kutiev et al. (2005) to collect a number of periods with increased geomagnetic activity. Hourly values of Kp and Dst were assigned to the data to support the analysis of the $r t$ and $b$ behavior. A large number of summary plots have been produced for the analysis, two samples of which are shown in Fig. 1. The left plot shows the period 14-17 July 2000 (Fig. 1(a)), while the right plot (Fig. 1(b)) shows the period 22-25 April 2001. The lower panel of each plot 


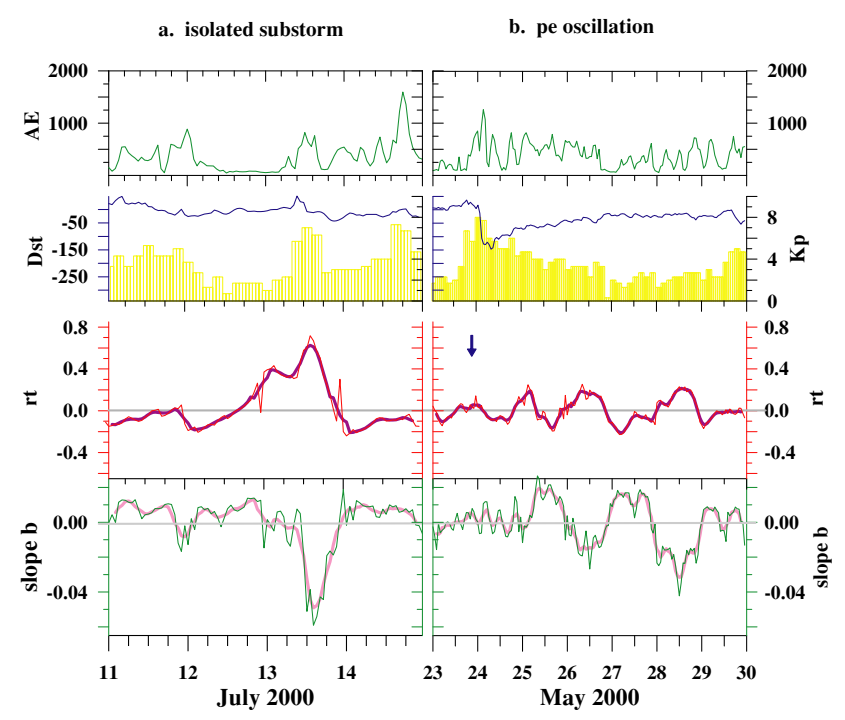

Fig. 2. The same as in Fig. 1, but for the periods 11-14 July 2000 (a) and 23-29 May 2000 (b).

shows $r t$ (red line, scaled on the left) and $b$ (green line, scaled on the right). The regression parameters $r t$ and $b$, averaged within a 5-h sliding window (heavy lines), are also plotted over the data. The smoothed variations (with heavier color lines) better visualize the main features appearing during the storms. The storm onset is shown by blue vertical arrows, and the duration is marked by green horizontal bars in the middle panels. The labels on the x-axes show the days of the respective month, with the numbers shown at the beginning of the day; these are the same for all panels of the column. The local time is $9 \mathrm{~h}$ ahead of UT. The next panel shows the geomagnetic indices: Kp as yellow vertical bars (scaled on the right) and Dst as a blue line (scaled on the left). The top panel represents the auroral AE index. The respective axis scales are the same in both rows of panels.

Figure 1(a) represents $r t$ behavior during an intense geomagnetic storm (Dst reaches -300 nT), starting with storm sudden commencement (SSC) time at 14:37 UT on 15 July 2000, when Japan was on the night side (00 LT). Figure 1(b) shows a storm with SSC at 16:01 UT on 21 April 2001, when Japan was on the dayside (07 LT). The onset of the first storm (denoted here as nighttime storm) is followed by an immediate decrease of $r t$, which continues until the area enters the dayside. During the day, a partial recovery takes place. During the following night and day, rt actually repeats its diurnal behavior from the previous period. In the second day, however, $r t$ recovers completely, which marks the end of the ionospheric storm. During the storm development, the slope $b$ tends to decrease slightly which, combined with the decrease of $r t$, shows that the negative disturbance is deeper towards the north. Figure 1(b) shows a moderate geomagnetic storms, with Dst gradually decreasing, reaching $-100 \mathrm{nT}$ at $13 \mathrm{UT}$ on the next day, 23 July. The indices AE and Kp show an substantial auroral activity. At the onset of the storm, $r t$ starts increasing and stays positive until the area enters the dark side. The slope $b$ also exhibits a positive deviation. The initial daytime enhancement of $r t$ is followed by the nighttime decrease of about
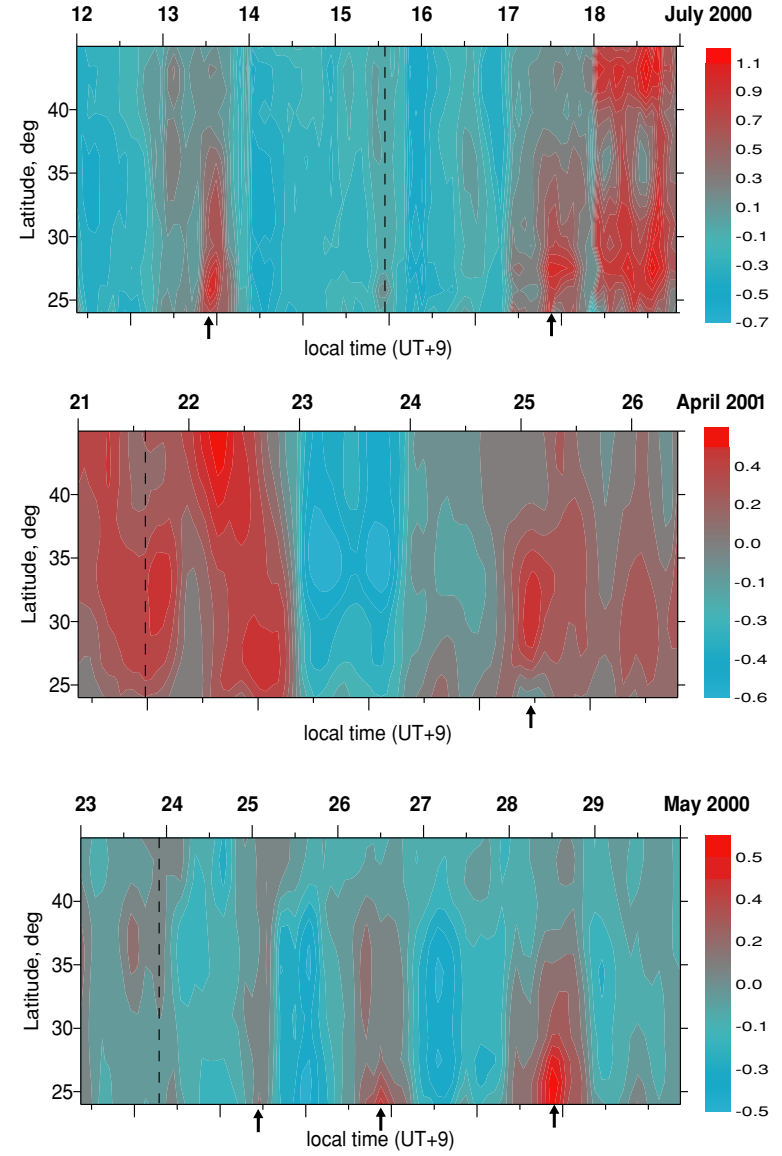

Fig. 3. Lat/UT maps of rTEC during storm periods of: $12-18$ July 2000 (a), 21-26 April 2001 (b) and 23-29 May 2000 (c). The magnitude of rTEC is coded by color scales on the right, as blue shows the negative values and red denotes the positive values. The storm onset is marked by a dashed vertical line; day numbers are shown on the top of each panel, placed at the beginning of each day. The ticks on the top axis show UT, while those on the bottom axis are shifted with $9 \mathrm{~h}$ to the left to show the local time; major ticks mark midnight.

$50 \%$. The negative disturbance stays stable during the following day and night, while the recovery takes place during the third day. At the end of the two storms, a visible increase in $r t$ with a sharp negative turning of $b$ (marked by the circled letters "pe") characterizes a persistent post-storm effect.

Figure 2(a) shows an enhancement of $r t$ associated with an isolated substorm on 13 July 2000. A large increase in $r t$-over $70 \%$ of the median level-occurs around $16 \mathrm{UT}$ on 13 July (22 LT). AE reaches $700 \mathrm{nT}$ and Kp increases to 7 , but with no visible decrease of Dst. Instead, the small positive peak of Dst indicates that the magnetosphere bow shock has been encountered by a solar wind magnetic cloud. At the time of largest increase of $r t$, the slope $b$ turns strongly negative, reaching a value of -0.06 . Both, $r t$ and $b$ recover on 14 July. The storm development can be followed in Fig. 1(a), where 14 July is also shown. A second substorm occurs at 0800 UT (17 LT) on 14 July with $\mathrm{AE}=1500 \mathrm{nT}$ and $\mathrm{Kp}$ reaching $7^{+}$. This substorm, however, does not produce a similar effect on $r t$ and $b$. Figure 2(b) shows a moderate storm with a SSC at 18 UT on 23 May 2000 , with a minimum Dst of $-150 \mathrm{nT}$; $\mathrm{AE}=1500 \mathrm{nT}$ and 


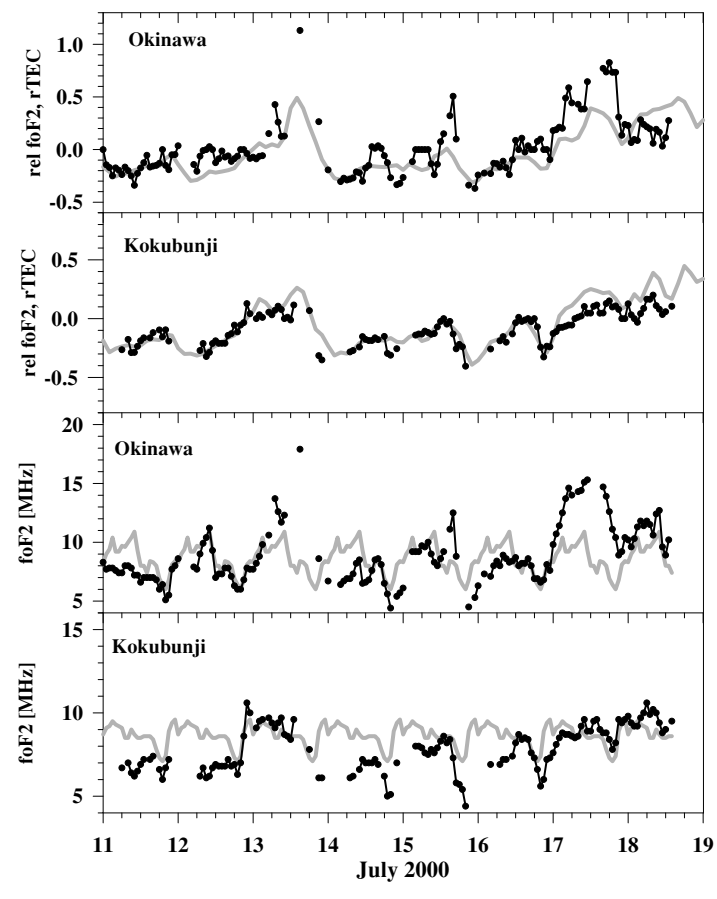

Fig. 4. foF2 variations (solid curves with dots) from the Kokubunji (bottom panel) and Okinawa (middle panel) ionosonde stations along with their respective monthly medians (thick gray curves) for the period 11-18 July 2000. The numbering on $\mathrm{x}$-axis of the lower panel represents UT for all of the panels above. The relative deviations for rfoF2 (solid curves with dots) and rTEC (thick gray curves), as slices of the rTEC contour plots at the Kokubunji and Okinawa latitudes, are compared in the top two panels.

$\mathrm{Kp}=8$ during the main phase of the storm. No visible change of either $r t$ or $b$ is seen during the storm. During the next four days, from 25 to 28 May, a well-expressed periodic variation of both $r t$ and $b$ is clearly observed. $r t$ and $b$ vary in anti-phase and change with an increasing period.

To study the post-storm effects shown in Figs. 1 and 2 in more detail, we supplemented $r t$ and $b$ plots with lat/UT maps of individual relative deviations rTEC and the variations in the $\mathrm{F}$ layer critical frequency foF2.

The lat/UT maps of rTEC provide a better insight into the actual topology of the disturbances and their development during geomagnetic storms. Figure 3 shows rTEC behavior during the same storms as those presented in Figs. 1 and 2, but resolved in latitude. All individual rTEC data from the cells within the central band are used in the Kriging spatial interpolation technique (SURFER 7.0; Golden Software, Golden, Colo.) for creating the maps. The color scale is given on the right of each panel, with the highest positive values in red and the lowest negative values in blue. The data of each hour represent a single vertical line. The storm onset, indicated with arrows on the first figures, is marked by a dashed vertical line in Fig. 3. The numbers of the days are shown at the top of each panel, along with the month and year (right end). The ticks of the bottom axis are shifted with $9 \mathrm{~h}$ to the left to show the local time; major ticks mark midnight. The top panel comprises the period of 12-18 July 2000 and includes both the isolated substorm and the major storm, presented separately on the left panels of Figs. 1 and 2. The structures of interest, the post-storm enhancements, are marked with arrows at the bottom axes. It should be noted that individual rTEC values are not corrected for the 27-day periodicity, as the latter was carried out for the regression mean $r t$. Therefore, rTEC maps can show larger extremes that exceed, on average, those of $r t$ by $30 \%$. The 27 -day periodicity and its two harmonics remove long-term variations - those longer than 9 days. For smaller size structures that appear on the maps for a day or two, the solar rotation periodicity will act as a shifted reference level; the structures will preserve their actual forms, but with a smaller amplitude. Another point of concern could arise from the Kriging interpolation procedure. The data points in each hour are of an order of 60 so that they sample sufficiently the latitude range $\left(24-45^{\circ} \mathrm{N}\right)$. The interpolation is made by using semi-variance functions, calculated separately for each particular map. Therefore, the interpolation reproduces the real shape of the map structures, but the amplitude of rTEC deviations should be viewed with caution.

In the July 2000 case (Fig. 3(a)), the rTEC enhancement on 13 July is associated with a moderate substorm $(\mathrm{AE}=700 \mathrm{nT}$ and $\mathrm{Kp}=7$ ). rTEC enhancement appears around $18 \mathrm{~h} \mathrm{LT}$ and disappears before sunrise. It stretches poleward up to $37^{\circ} \mathrm{N}$. A negative disturbance takes place after sunrise, staying throughout the whole latitude range until the onset of the storm on 15 July. The nighttime storm produces a negative disturbance, which disappear in the morning local time on 17 July. After sunset, a strong enhancement of rTEC takes place at latitudes below $37^{\circ} \mathrm{N}$, with the strongest appearing between $27^{\circ}$ and $30^{\circ} \mathrm{N}$. Shortly after that, the enhancement starts to decrease from its equatorial end, literally tearing off the place of origin. This enhancement disappears with sunrise. Another strong daytime increase in rTEC on 18 July appears simultaneously throughout the whole latitudinal range, with a distinct minimum doublet around $35^{\circ} \mathrm{N}$. This latter event is associated with a weak substorm $(\mathrm{AE}=600 \mathrm{nT})$ that occurred around $06 \mathrm{UT}$.

Figure 3(b) provides details on the storm development shown on Fig. 1(b). The storm of 22-25 April 2001 starts with SSC at 16 UT on 21 April, but develops gradually. The minimum Dst of $-100 \mathrm{nT}$ is reached at $13 \mathrm{UT}$ on 22 April (22 LT), at the time when $\mathrm{AE}=1000 \mathrm{nT}$ and $\mathrm{Kp}$ increases to 7. At the storm onset, a substantial increase of TEC at lower latitudes is observed, which is probably a post-storm effect of a previous geomagnetic storm that occurred on 18 April 2001 (not shown here). The present storm starts producing a marked enhancement of rTEC at the beginning of 22 April, when Japan actually enters the daytime. This enhanced structure is tilted to earlier hours, which means that the positive disturbance propagates southward. Following this daytime increase, a negative disturbance starts to appear from the north with the entering darkside. This disturbance occupies the entire latitude range on 23 April, with a minimum at approximately $35^{\circ} \mathrm{N}$. The post-storm increase of rTEC, which is part of the equatorial crest, can be seen as a small wedge at $25^{\circ}$ latitude as early as at 06 UT (15 LT) on 24 April, increasing markedly around noon of 25 July. The latter structure exhibits the same behavior as that in the July 2000 case: it tears off the equatorial crest region. The 


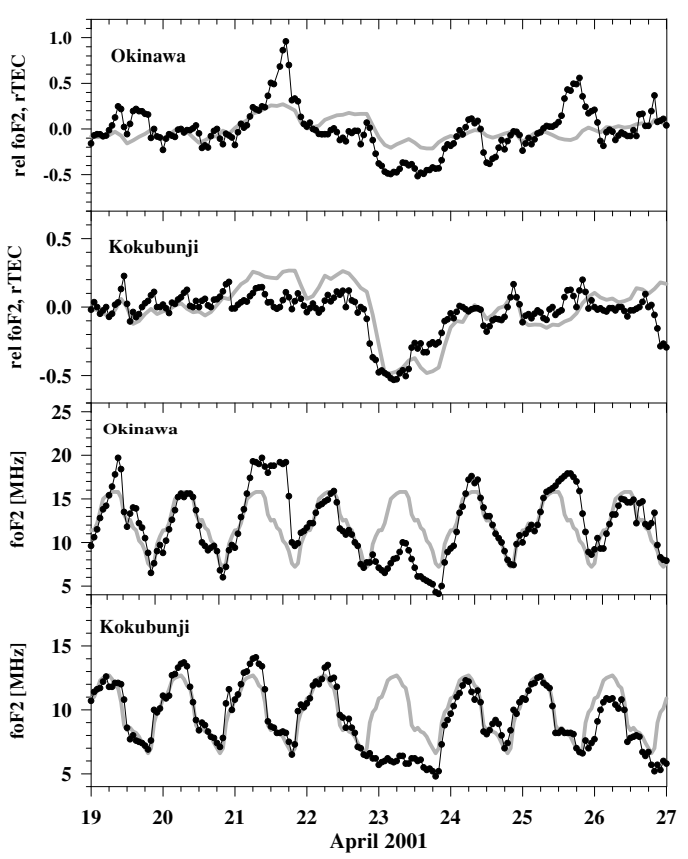

Fig. 5. The same as in Fig. 4, but for the period 19-26 April 2001.

maximum of this structure stretches between $27^{\circ}$ and $35^{\circ}$ latitude. Enhanced rTEC, also observed on the next day, 26 April, resembles that of 25 July with respect to shape.

A series of three post-storm features (bottom panel) with increasing rTEC amplitude appear between 25 and 28 May 2000 , following the weaker storm on 23 May. The poleward expansion does not show the 24-h periodicity shown by the change of the slope, but the enhancement is alternatively replaced by depression, with no diurnal dependence.

The $\mathrm{F}$ region behavior, represented by foF2 from two Japanese ionosonde stations, Kokubunji (35.7N, 139.5E) and Okinawa $(26.3 \mathrm{~N}, 127.8 \mathrm{E})$, is presented in the next Figs. 4-6 for the same storms shown above. Kokubunji (KOK) has a geomagnetic latitude of $26^{\circ} \mathrm{N}$, while Okinawa (OKI) is at about $10^{\circ}$ equatorward. Figure 4 shows foF2 variations (black curves with dots) from KOK (bottom panel) and OKI (middle panel) for the period 11-18 July 2000 , along with their respective monthly medians (thick gray curves). The upper two panels show a comparison of the relative deviations of foF 2 from medians (rfoF2), as calculated by the same type of expression as Eq. (1), with curves (thick gray), presented as slices of the rTEC contour maps of Fig. 3 for the latitudes of KOK and OKI. The curves in the figure are drawn discontinuously in order to see the gaps in the data; consequently, single data points between gaps are shown by dots only. The figure covers the periods shown in Figs. 1(a) and 2(a). The negative disturbance of $r t$, seen on 15 and 16 July 2000, can be traced in the foF2 and rfoF2 variations, although the large gaps of ionosonde data make a detailed comparison difficult. On 17 June, when $r t$ and $b$ exhibit a marked poleward expansion of the crest region, the $\mathrm{F}$ layer reacts in a very distinct way. No visible change is seen at KOK, while at OKI, only $10^{\circ} \mathrm{S}$, a large positive deviation of foF2 takes place that exceeds the median level by $80 \%$. The large poleward expansion during the isolated substorms on 13 July 2000 (shown in

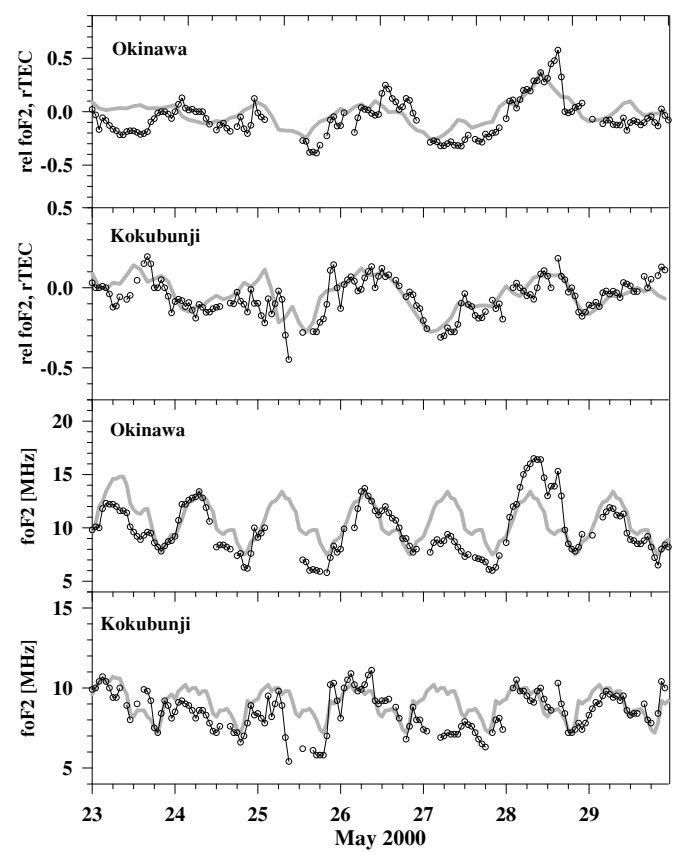

Fig. 6. The same as in Fig. 4, but for the period 24-30 May 2000.

Figs. 2(a) and 3(a)), can also be traced in Fig. 4. The single point at 15 UT shows an increase of $113 \%$ over the median, although the gaps on both sides of this measurement do not allow the duration of this increase to be determined. The contour slice of rTEC at OKI does not show such large enhancements as foF2. At $15 \mathrm{UT}$ on 13 July, rTEC shows an increase of $50 \%$ against the $113 \%$ increase shown by rfoF 2 ; on 17 July, the rTEC increase is around $40 \%$ as compared to the $70 \%$ increase in the rfoF2. A similar discrepancy can also be seen around 16 UT on 15 July. (See following section for a discussion of the degree of agreement between both quantities and the possible cause of the observed discrepancies.) At Kokubunji, however, both rfoF2 and rTEC variations are in a good agreement throughout the entire study period.

The daytime storm of 22-25 April 2001 in Figs. 1(b) and 3(b) can be compared with the foF2 behavior shown on Fig. 5. The storm onset at around 01 UT on 22 April has no effect on the F layer density during the whole day of 22 April, while $r t$ at the same time shows an increase that exceeds $20 \%$. Note that the $\mathrm{X}$-axis indicates UT time; local day starts 9 earlier and can be recognized from the diurnal variations of medians. At sunrise on 23 April, a large negative disturbance takes place at both stations that lasts the entire day. As the AE index shows, an intense substorm activity takes place during that day, generating a composition bulge that enters the area at sunrise and stays there during the day. Moreover, at sunrise on 23 April, the deviation of foF 2 from the median at KOK begins about $2 \mathrm{~h}$ earlier than that at the OKI latitude. This is obviously the time needed for the equatorial edge of the composition bulge to shift $10^{\circ}$ southward. The contour slice rTEC and rfoF2 show that the negative disturbance is deeper at the latitude of KOK than at that of OKI. The following day, 24 April, the negative disturbance recovers and while at $\mathrm{KOK}$ foF 2 is close to median values, a $10 \%$ increase at $\mathrm{OKI}$ is visible around noon local 
time. The maximum "pe" effect is observed on 25 July, when both $r t$ and foF 2 at OKI show a $60 \%$ increase, while the sliced rTEC does not increase at all. In contrast, both rfoF2 and rTEC at KOK do not show any visible increase on 25 July. The contour plot of Fig. 3(b) shows that the maximum of rTEC occurs from $26^{\circ}$ to $35^{\circ}$ latitude, which falls between the locations of OKI and KOK. It is probable that the mapping procedure producing the rTEC surface on Fig. 3(b) smoothes out the latitude edges of this structure, resulting in a lack of increase in the rTEC slices at the latitudes of KOK and OKI.

Figure 5 shows a large increase in foF2 at OKI station on 21 April that reaches $96 \%$ at 17 UT compared to the $20 \%$ increase in rTEC. In comparison, this effect is less than $10 \%$ at KOK. This enhancement does not seem to be associated with geomagnetic activity, but could be a "pe" effect of a weak geomagnetic storm that occurred on 19 April 2001.

As already mentioned, an oscillation-type behavior is observed during the May 24, 2000 storm that resembles the "pe" effect observed in the previous figures. Following the geomagnetic storm on 24 May 2000, three negative disturbances occur at both stations on 24 (very weak), 25 (strong) and 27 (strong) May, as seen from Fig. 6. The ionosphere recovers between these negative disturbances. On 28 May, a marked increase of $60 \%$ is seen at OKI station. This behavior is similar to that of the $r t$ and $b$ shown in Fig. 2(b). The slope $b$ varies in anti-phase with $r t$, exhibiting an oscillation-type variation from 25 to 28 May, changing its sign alternatively every $24 \mathrm{~h}$. Figure 3(c) shows that these oscillations are structures with increased and decreased rTEC seen from the lowest latitude up to $40^{\circ} \mathrm{N}$. The increased fraction obviously originates from the equatorial crest region, while this is not certain for the negative fraction. The increased fraction on 26 and 28 May appear in the evening hours local time. The interpolation procedure may produce an effect that closes up the contours at the borders of the map area.

\section{Discussion}

Figures 1 and 2 show a substantial increase in $r t$ and strong negative turning of $b$ at the end of geomagnetic storms. This coherent behavior of $r t$ and $b$ is a persistent feature appearing at the end of the recovery phase of almost all of the storm periods examined and is an effect of the data treatment. Figure 3 makes the interpretation clearer. During the poleward expansion of the equatorial crest region, the slop turns strongly negative and $r t$ increases, mainly due to the contribution from the southern part. In this respect, the regression parameters actually augment the differences between the mid-latitude and equatorial parts and make the effect of poleward expansion more pronounced. The lat/UT maps provide a detailed picture of the latitudinal variations in the observed phenomena. It should be noted that the latitude profiles composing the maps are actually tilted $45^{\circ}$ to the east. Therefore, if some structures propagate during the periods examined and their velocity has a zonal component, the maps will present the movement as a shift with time. This comparison of TEC variations with those of the critical frequency foF 2 provides a picture of how synchronously the $\mathrm{F}$ region and the total ionization behave.

The regression parameters $r t$ and $b$, the lat/UT maps and foF 2 are available for studying the post-storm behavior of ionosphere in middle and low latitudes during the three periods considered: 11-18 July 2000, 22-25 April 2001 and 23-29 May 2000. These periods are discussed separately in the following sections.

\section{$4.1 \quad 12-18$ July 2000}

Figure 2(a) shows that a strong $r t$ enhancement appears in association with an isolated substorm that occurs during the night, outside of geomagnetic storms. The large AE and $\mathrm{Kp}$ in this case imply that the Joule heating in the auroral oval is sufficient to produce an composition bulge with a subsequent negative disturbance at mid-latitude. Even when this happens, the strong expansion of the low latitude ionosphere obviously dominates over the northern effect. The large nighttime expansion of equatorial crests is an effect of either the upward lifting of the equatorial ionosphere by the $\mathrm{E} \times \mathrm{B}$ drift or the presence of the eastward electric field. One possible explanation is that proposed by Kelley et al. (2004) for the formation of SED. Indeed, the ionization during the whole day of 13 July seems to be enhanced, especially around noon local time, as indicated by foF 2 in Fig. 4 and $r t$ in Fig. 2(a). This implies an action of upward $\mathrm{E} \times \mathrm{B}$ drift, which lifts the equatorial ionization at a higher altitude and fills it in afterwards at the mid-latitude $F$ region. From the nightside onwards, a poleward electric field drives the $\mathrm{F}$ region ionization westward and accumulates it at the dusk sector. At the termination of dusk, the upward drift from the dayside and the zonal drifts from the nightside form the so-called "evening vortex" (Eccles et al., 1999; Heelis, 2004). This combined drift may shift rTEC enhancement slightly into the nightside, which is what is actually what is seen in Fig. 3(a). It remains unclear, however, how an eastward electric field penetrates the daytime ionosphere as early as 00 UT on 13 July, several hours before $\mathrm{Kp}$ and $\mathrm{AE}$ increase. One possible explanation of this fact is that the daytime enhancement on 13 July can be a post-storm effect of the moderate substorms that occurred on 5-6 July.

The ionospheric behavior during the storm of 15 July 2000 has been studied by Vlasov et al. (2003) using TEC and satellite data. They observed a strong increase in TEC in the American sector that was associated with a sharp decrease in Dst and the penetration of intense electric field to low latitudes. At the time of storm commencement (14:37 UT), Japan is at midnight local time. In contrast to the American sector (at the storm onset the local time there is around 08), only a slight, short-lived increase is observed at all latitudes in the Japan sector, followed by a negative disturbance during the next $24 \mathrm{~h}$. The slight increase appears again at midnight on 16 July, and on 17 July, only more pronounced in the latter case. The latter structure we identify as a poleward expansion effect. The poleward expansion starts in the late evening hours and forms a maximum around $27^{\circ} \mathrm{N}$. Shortly before midnight, the rTEC south of the maximum starts to decrease, and the structure visibly tears off the crest region. The equatorial crests seem to shrink inwards, probably due to a westward electric field. In this movement, the low latitude ionization may 
remain unchanged or even increase due to the supply from higher altitudes where the downward $\mathrm{E} \times \mathrm{B}$ drift squeezes the plasma in magnetic flux tubes down to lower altitudes. Liu et al. (2002) used the Fejer and Scherliess (1997) empirical model of the equatorial zonal electric field driven by the $\mathrm{AE}$ index to calculate the vertical drift during the poststorm increase. The model did not predict any $\mathrm{E} \times \mathrm{B}$ drift on 17 and 18 July 2000.

The enhancement of rTEC observed on 18 July 2000 in Fig. 3(a) is even more peculiar. It is difficult to judge whether this enhancement, seen throughout the day, is a further development of the poleward expansion event discussed above, although it occurs again $24 \mathrm{~h}$ later. If we look back to the previous day, 17 July, we will see that the same type of enhancement has occurred, but that the increase in the northern part is less than $10 \%$. Even the small forklike doublet seen around $33^{\circ} \mathrm{N}$ resembles the minimums of rTEC on 18 July. AE during that day does not exceed 600 $\mathrm{nT}$, so the low geomagnetic activity makes the penetration of external electric fields quite unlikely. The strong rTEC enhancement, both at the low latitude part and the midlatitude part, exceeds $100 \%$ over the median values. As was mentioned above, the amplitude of rTEC deviations contain a 27-day periodicity, in addition to that invoked by the current conditions. The latitude average $r t$ value on 18 July (this day is not shown on Fig. 1(a) is around 40\%, indicating that the rTEC increase is still significant.

\subsection{1-25 April 2001}

The strong enhancement of rTEC on Fig. 3(b) increases on 22 April, when the local time is 09 hours in the morning. The enhanced structure is slightly tilted, which means that the positive disturbance propagates southward. The tilt measured between $45^{\circ}$ and $30^{\circ}$ latitude is equivalent to propagation with a speed of $188 \mathrm{~km} / \mathrm{h}$ or $52 \mathrm{~m} / \mathrm{s}$. This velocity is much slower than that of a large traveling ionospheric disturbance (TID) generated during the initial phase of the storms (Tsugawa et al., 2004) and probably can be attributed to the changes in thermosphere composition. The positive disturbances may arise when the composition bulges from the summer and winter hemisphere collide at the winter side (Field and Rishbeth, 1997) or they may occur by uplifting of the $\mathrm{F}$ layer by the meridional winds (Namgaladze and Förster, 2000). Below $32^{\circ} \mathrm{N}$, the positive disturbance is not tilted; the maximum effect is observed between $26^{\circ}$ and $30^{\circ}$ latitude. The large latitudinal gradient exists between ionosondes OKI and KOK, as seen from Fig. 5. At OKI, the positive deviation of rfoF2 reaches $110 \%$, while that at KOK is negligible. The negative disturbance, which follows on 23 April, is probably produced by a composition bulge with an increased molecular fraction. At $45^{\circ} \mathrm{N}$ it becomes visible around $06 \mathrm{LT}$ and probably appears earlier at higher latitudes as a typical nighttime storm (Muhtarov and Kutiev, 1998). At lower latitudes the negative disturbance propagates much faster. Two minimums are seen at around $35^{\circ} \mathrm{N}$ : the first at 04 UT on 23 April and the other 12 hours apart. A partial recovery takes place between the two minimums, which is more pronounced from the north and from the south. The southern recovery with a wedge-like shape in the afternoon hours local time appears again the next day, 24 April, at the same local time. It obvi- ously presents a poleward expansion of the equatorial crest. On 25 April, this small wedge-like structure is replaced by a marked increase of rTEC, separated from the equatorial crest. The structure is similar to that on 17 July 2000, shown on the upper plot. The structure of 25 April is reproduced once again on 26 April, but with a lower amplitude in the maximum part .

\subsection{3-29 May 2000}

Figure 2(b) shows a rare case of a moderate geomagnetic storm with a small effect on ionosphere in which a series of positive and negative disturbances alternatively take place with slightly increasing period. The slope $b$ is changing synchronously in anti-phase. Starting from 00 UT on 25 May 2000, negative and positive disturbances alternatively change during the next four days. The positive disturbances originate from the equatorial crest. While the first increase of rTEC on 25 May happens around noon local time, the other two features are nighttime phenomena, starting in the dusk sector. Their shapes resemble that of the substorminduced enhancement on 13 July 2000.

Special attention should be stressed on the joint use of foF2 and TEC derivatives in the present analysis. TEC in this paper is a quantity that is averaged over single measurements (30-s sampling rate) within an area $1.5^{\circ} \times 1.5^{\circ}$ and 1-h time frame; rTEC is its relative deviation from a 27-day median. The regression parameters $r t$ and $b$ represent the latitudinal distribution of rTEC by approximating it with a planar surface. Contrary to these averaged quantities, foF2 (respectively rfoF2) is an instant measurement of plasma frequency at the peak of the F layer. In the presence of small-scale disturbances or variations with less than $1 \mathrm{~h}$ on the time scale, foF 2 and TEC (rfoF2 and rTEC) may differ significantly, depending on amplitudes of these variations. We believe that this is the explanation for the large differences between rfoF2 and sliced rTEC in upper two panels of Figs. 4-6. To estimate the degree of coherence between rfoF 2 and rTEC behavior, we calculated their cross-correlation functions separately for the KOK and OKI stations, based on the data from the three figures. As expected, the correlation is higher at Kokubunji than at Okinawa. At time lag $=0$, the cross-correlation function (equal to the correlation coefficient) at Kokubunji is 0.768 and at Okinawa, 0.608. It is interesting to note that the maximum cross-correlation at Okinawa is obtained at the time lag $=-1$ which means that Okinawa foF2 reacts $1 \mathrm{~h}$ earlier that the averaged TEC.

If we summarize the properties of the TEC enhancements discussed above, we have to mention first that they appear outside of the initial and main phases of geomagnetic storms, at a time when the convection electric field from auroral oval usually penetrates deep into the low latitudes and equatorial ionosphere and produces electromagnetic drifts. The TEC enhancement considered here occurs in periods of low magnetic activity that are characterized by Dst and Kp indices. The AE index, issued by the WDC for Geomagnetism, Kyoto, was used to define the substorm activity. Most of the enhanced TEC events appear in late afternoon hours local time and develop after sunset. These could be satisfactorily explained by the mechanism proposed by Kelley et al. (2004) for SEDs, if the intense electric field 
penetrates to low latitudes at that time, but this is not evident. The nighttime zonal neutral wind can also create a polarization electric field (disturbance dynamo), which consecutively can drive the ionization in the same direction. These polarization fields have their effect at night; during the daytime the high conductive E layer causes them to disappear. The post-storm events seen on 18 July 2000 and 25-26 April 2001 are obviously daytime phenomena that cannot be produced by the zonal winds. Meridional winds blowing across the equator may transport ionization from the summer to winter crest regions and probably can produce the effect of poleward expansion observed on our data. Such an effect of strong asymmetry of equatorial crests has been reported by Lin et al. (2005).

\section{Conclusion}

The data set, based on data collected from the dense GPSTEC network in Japan, has been modified by removing diurnal, seasonal and solar rotation induced variations. The modified data are considered to be representative of that part of the variations induced by geomagnetic activity only. This allows several phenomena to be revealed that occur outside of the geomagnetic storms, denoted in the paper as post-storm effects. In all of the cases examined, with one exception, the geomagnetic and auroral activity was low. The main results can be formulated as follows:

1. Most of the post-storm TEC enhancements are part of the equatorial crest region and extend poleward during the late evening and nighttime hours. In some cases, the enhanced TEC structures develop by separating from the crest region.

2. Daytime TEC enhancements are also observed. Their structures are not confined to the equatorial crests region, but occupy the whole of the latitude range considered in this study.

3. TEC post-storm enhancements are generally in agreement with those seen in foF 2 variations.

Acknowledgments. This work was carried out during the stay of one of the authors (Ivan Kutiev) as visiting professor at the SolarTerrestrial Environment Laboratory (STEL) of Nagoya University.

\section{References}

Codrescu, M., T. J. Fuller-Rowell, and I. Kutiev, Modeling the F-layer During Specific Geomagnetic Storms, J. Geophys. Res., 102, 1431514320, 1997.

Eccles, J. V., N. Maynard, and G. Wilson, Study of the evening plasma drift vortex in the low-latitude ionosphere using San Marco electric field measurements, J. Geophys. Res., 104, 28133-18145, 1999.

Fejer, B. G. and L. Scherliess, Empirical models of storm time equatorial zonal electric fields, J. Geophys. Res., 102, A11, 24047-24056, 1997.

Field, R. P. and H. Rishbet, The response of the ionospheric F2-layer to geomagnetic activity: an analysis of worldwide data, J. Atmos. SolarTerr. Phys., 59(2), 163-180, 1997.

Heelis, R. A., Electrodynamics in the low and mid latitude ionosphere: a tutorial, J. Atmos. Solar Terr. Phys., 66, 825-838, 2004.

Kelley, M. C., M. N. Vlasov, J. C. Foster, and A. J. Coster, A quantitative explanation for the phenomenon known as storm-enhanced density, Geophys. Res. Lett., 31, L19809, doi:10.1029/2004GL020875, 2004.

Kutiev, I., P. Muhtarov, and P. Bradley, Penetration of ionospheric disturbances into the European region during geomagnetic storms, Adv. Space Res., 22(6), 865-867, 1998.

Kutiev, I., S. Watanabe, Y. Otsuka, and A. Saito, Total electron content behavior over Japan during geomagnetic storms, J. Geophys. Res., 110(A1), A01308, doi:10.1029/2004JA010586, 2005.

Lin, C. H., A. D. Richmond, J. Y. Liu, H. C. Yeh, L. J. Paxton, G. Lu, H. F. Tsai, and S.-Y. Su, Large-scale variations of the low-latitude ionosphere during the October-November 2003 superstorm: Observational results, J. Geophys. Res., 110, A09S28, doi:10.1029/2004JA010900, 2005.

Liu, L., W. Wan, B. Ning, H. Yuan, and J. Y. Liu, Low latitude ionospheric effects near $120^{\circ} \mathrm{E}$ during the great geomagnetic strom of July 2000 , Sci. China (series A), 45 (suppl.), 148-155, 2002.

Liu, L., W. Wan, C. C. Lee, B. Ning, and J. Y. Liu, The low latitude ionospheric effects of the April 2000 magnetic storm near $120^{\circ} \mathrm{E}$, Earth Planets Space, 56, 607-612, 2004.

Muhtarov, P. and I. Kutiev, Empirical modelling of ionospheric storms at midlatitudes, Adv. Space Res., 22, 6, 829, 1998.

Muhtarov, P., I. Kutiev, and L. Cander, Geomagnetically correlated autoregression model for short-term prediction of ionospheric parameters, Inverse Problems, 18(1), 49-65, 2002.

Namgaladze, A. A. and M. Förster, Analysis of the positive ionospheric response to a moderate geomagnetic storm using a global numerical model, Ann. Geophys., 18, 4, 461-477, 2000.

Otsuka, Y., T. Ogawa, A. Saito, T. Tsugawa, S, Fukao, and S. Miyazaki, A new technique for mapping of total electron content using GPS network in Japan, Earth Planets Space, 54, 63-70, 2002.

Tsugawa, T., A. Saito, and Y. Otsuka, A statistical study of large-scale traveling ionospheric disturbances using the GPS network in Japan, $J$. Geophys. Res., 109, A06302, doi:10.1029/2003JA010302, 2004.

Vlasov, M., M. C. Kelley, and H. Kil, Analysis of ground-based and satellite observations of $\mathrm{F}$ region behavior during the great magnetic storm of July 15, 2000, J. Atmos. Solar Terr. Phys., 65, 1223-1234, 2003.

I. Kutiev (e-mail: ivan.kutiev@geophys.bas.bg), Y. Otsuka, A. Saito, and $\mathrm{S}$. Watanabe 